\title{
Trends, Technologies, and Future Challenges in Wireless Sensor Network's
}

\author{
Putri Kevin \\ Department of Computer Science, \\ Brahijaya University, \\ Indonesia \\ lettylord@yahoo.com
}

\author{
Dian Viely \\ Department of Computer Science, \\ Brahijaya University, \\ Indonesia
}

\begin{abstract}
Wireless microsensor is the most significant and innovative technology in the $21^{\text {st }}$ Century. The development of sensor networks is examined, and applications like infrastructure security, monitoring ecosystem, and traffic management. The technical difficulties in sensor network development include routing, network exploration, signal processing, and security. This paper concludes by exploring results from different research in sensor network algorithms, localized algorithms and directed diffusion, distributed tracking in wireless ad hoc networks, and distributed classification using local agents.
\end{abstract}

\section{Keywords}

Sensor network, Localization, RSSI, Ad-hoc, Anchor

\section{INTRODUCTION}

Cheap and smart devices with multiple onboard sensor networks through a channel and implemented on a large scale provide unrivalled opportunities for smart homes, cities, and the environment. In defense surveillance and reconnaissance, detect and track the incoming threats. Each sensor node has multiple onboard sensors with processing capabilities operating in acoustic, seismic, infrared (IR), magnetic modes, image recognition, and micro radars as shown in Table 1. Table 1, summarizes the spectrum of potential attributes in general networks of sensors [13].

\section{HISTORY OF RESEARCH IN WSNS}

For the research and development of sensor, three different researches technology is required: Sensing, communication and computing (software and hardware) [4].

\subsection{Early Research in Military}

In the cold war, an acoustic sensor known as a sound surveillance system (SOSUS) under the ocean was deployed to detect and track the Soviet submarines quietly. SOSUS is now used by the NOAA (National Ocean-Atmosphere Administration), e.g., seismic and aquatic life. Airborne Warning and Control System (AWACS) planes and air defence radar systems are deployed to protect the USA and Canada. In general, these sensor networks follow a hierarchical processing system where processing occurs at consecutive stages until the information reaches the user; in most cases, humans play an important role in the system [5-7].

\subsection{Distributed Sensor Program in Defense}

Modern research on DNS started in 1980 by DARPA. It was assumed that local low-cost distributed networks communicate with one another but autonomously operate with information routed to that node that best uses this information. In 1978 components for DNS were identified in distributed sensor nets [8]. It included the sensors, communication system (protocols), processing techniques, and algorithm. DARPA was sponsoring AI research for understanding signals, distributed problem-solving techniques, and assessing conditions. Few technology components were available off-shelf. The resulting DNS had to deal with distributed computing support and signal processing, tracking Distributed acoustic system was chosen for demonstration as a target problem. They innovated a communication-oriented operating system known as accents, whose first support is device configuration, rebinding, and transparent network. Accent has evolved into the Mach operating system, which has found significant market adoption. CMU activities included network protocol to support dynamic binding of active communication measures. An interface language for building distributed software, dynamic load balancing, and error reconfiguration system for DSN software [9-11]. MIT researchers focused on detecting and tracking helicopters using an acoustic microphone array by signal matching and abstraction techniques. Signal abstraction means a signal with multiple levels. Higher-level acquired by compressing information in the lower level. They offer a computational structure for thinking about signal processing processes that mimic what people use as they interactively process and view real-world signals. In addition, MIT has also created the Signal Processing Language and Interactive Computation Environment (SPLICE) for DSN data analysis and algorithm creation, and Pitch Director's Assistant for the Interactive computation of fundamental Frequency using domain Information [12-15]. In the 1980s, Advanced Decision Systems (ADS), Mountain View, CA, developed a multi-hypothesis monitoring algorithm to deal with challenging circumstances, including high target density, missed detections, false alarms, etc. distributed implementation was decomposed. For demonstration purposes, the MIT Lincoln Laboratory built a real-time testbed for low-flight aircraft acoustic detection. The nodal machine (for target tracking) consists of three MC68000 processors with $256 \mathrm{kB}$ memory, $512 \mathrm{kB}$ shared memory, and a custom operating system. Ethernet and the microwave radio used for communication. This is demonstrated in figure 1 below. The DSN testbed was verified by low-flying 
aircraft, which were successfully tracked with acoustic sensors and TV cameras [16-19].

\subsection{Military Sensor Networks in 80s}

Sensor network becomes a key component of centric warfare. Sensors are mounted on weapons that are controlled by a separate platform. They collaborate over a communication network, and information is sent to the shooter and tracking performance through multiple observations, response time, extended range, and geometric and environmental diversity. US navy developed the Cooperative Engagement Capability (CEC) system with multiple radars to collect air targets data. Since all the nodes have access to the same information, a "common operating picture" is necessary for consistent military operations. Some military sensor networks include acoustic sensor arrays Anti-submarine warfare such as FDS (Fixed Distributed System), ADS (Advance Deployable System), Unattended Land Sensors (UGS), Remote Battlefield Sensor System (REMBASS), and the Tactical Remote Sensor System (TRSS) [20-23].

\subsection{Sensor Era in $21^{\text {st }}$ Century}

Cheap and small sensors based on MEMS technology, wireless network, and low power processor allow ad-hoc wireless networks with numerous applications. DARPA started developing a new network and implementation of sensor nodes in the ad hoc network pattern. Today's networking strategies, built for voice and data, and relying on a fixed infrastructure, would not be enough for battlefield use. The second research was network information processing, which means extract useful and timely data from sensors [24-27]. SenIT developed a network with the new specification. The network is interactive and programable. Software and overall device design help low latency, energyefficient operation, built-in autonomy and longevity, and low possibility of detecting operation. A network of SensIT nodes can enable the detection, recognition, and tracking of the target, both within the network and outside the network, such as an overhead asset.

\section{TECHNOLOGY TRENDs}

Local companies like an ember, crossbow, and sensorial developing sensors node. Personal digital assistants (PDAs) using Palm or Pocket PC operating systems provide considerable processing and computational power in a small kit. Wireless networks based on IEEE 802.11 standard have bandwidth nearly equal to a wired network. IEEE 802.15 standard for PAN with a radius of $5-10 \mathrm{~m}$. short-range sensor network encourages to implement in PAN fashion. Further-Increased chip performance and processor production capacities have lowered the energy per bit demand for both computation and communication. Sensing communication and processing can be done on a single-chip now a day. The University of California Berkeley in 1990 developed a MEMS sensor that was small enough to best fit into a cubic millimeter. Smart Dust sensors can be deployed using a 3x10 mm "wavelet" shaped like a maple tree seed and lowered to the ground Table 2.

\section{TRACKING AND LOCALIZATION}

MEMS IC technology is drastically progressing now a day along with wireless communication. Connecting multiples sensors to a network from WSN[1]. The sensor reads the physical characteristic like temperature, pressure, and distance, etc [28]. WSN helps in health monitoring of patient health, military targets with location recognition. In localization, collected data from the sensor node is useless if the location is not precise. To fix this localization problem, equip each sensor node with GPS, which is practically impossible because of cost and power consumption increase. GPS glitch in dense forest, underground and indoor. Self-localization method sensor node used localization protocol to estimate position. Beacon nodes are used to estimate location in this protocol. Blind nodes provide information about the position of non-beacon nodes, known as a blind node. Blind nodes use information messages of beacon to locate his position [29]. All localization schemes consist of two phases: 1) distance/angle approximation; 2) position computation. The most distance/angle approximation techniques used to approximate distance or angle between two sensor nodes are Time Difference of Arrival (TDOA), Time Of Arrival (TOA), Received Signal Strength Indicator (RSSI), Angle Of Arrival (AOA), and Hop-count. In position computation, the unknown node's position is estimated based on the available information of distance or angle and references nodes' positions [30]. The commonly used techniques include lateration, triangulation, bounding box, probabilistic approach, and fingerprinting.

\subsection{Localized Algorithms and Distributed Diffusion}

In a localized (or distributed) algorithm, sensor nodes communicate with the adjacent sensor. Localized algorithms are robust to node failure and network changes. Communication costs also go along with rising network size. However, localized algorithms are challenging to design because of the complex relationship between local behaviour and global behaviour. The local algorithm may not perform better than the international [31]. Direct diffusion is belonging to the localized algorithm. Diffusion is a type of broadcast routing that does not prescribe the address of the destination node. Packets are forwarded to adjacent nodes, and a direction or gradient is overlaid to control the transfer or forwarding of the packet, which arrived at the destination. The gradient may depend on geographic information, power, congestion, and other resources of a network node. Intermediate nodes can transfer data locally to improve performance, robustness, and scalability. Simulation and experimental findings of direct diffusion in sensor networks suggested that multicast protocols (an IP-based multicast routing technique) require less than half of the energy needed for flooding, and diffusion needs just $60 \%$ of the energy for multicasting. These savings are accomplished by removing paths expended delivering redundant data and in-network consolidation, such as intermediate nodes suppressing duplicate position estimates [32].

\subsection{Distributed Tracking in Wireless Ad-Hoc Networks}

Sensors should communicate and exchange data for exploiting the advantages of data fusion of the sensor, but this should be achieved without submitting data requests and gathering data from all sensors, thus overloading the network and consuming energy resources. The ad hoc networks with microsensors face various challenges due to communications and processing. Target tracking has been extensively studied for the sensor node wide networks, and distributed tracking algorithms are available [33]. In the distributed tracking system, the problem of the dynamic sensor network. The approach to information-driven sensor querying (IDSQ) has been developed, allowing cooperation based on resource constraints and the cost of transmitting the information. Each sensor measures the estimated information utility of a piece of non-local sensor data and uses this measure to decide from which sensor the data is requested. The information utility 
functions used include entropy, Mahala Nobis distance, and the predicted post-distribution measure. A data association is required in multiple targets tracking close to each other relative to sensor measurement error. It is computationally expensive to implement on an ad hoc network.

\subsection{Distributed Classification in WSN Using}

\section{Mobile Agents}

In the sensor network, data is collected by the sensor and transmitted to centre nodes for processing. Usually, the sensor's bandwidth is smaller than the wire, so the network communication requirement exceeds. Mobile agents suggested to this problem. In a mobile-based agent in DNS, data stay at the sensor while fusion code is moved to data. If the agent is smaller than the data, the bandwidth requirement may be shorter. A sensor network is scalable, and performance is not affected by the number of sensors in a network.

Table 1: A Sensor Layout and Architecture

\begin{tabular}{|l|l|}
\hline Sensors & $\begin{array}{l}\text { Size: MEMS large (Radar, Satellite) } \\
\text { Number: Small, Large } \\
\text { Type: Passive (Acoustic, IR, Magnetic, Video), Active (Radar, Lidar) } \\
\text { Spatial Coverage: Dense and Spare } \\
\text { Deployment: Fixed and Planned, Ad-hoc } \\
\text { Dynamics: Static and Mobile }\end{array}$ \\
\hline Sensing Entities of Interest & $\begin{array}{l}\text { Extent: Distributed Localized } \\
\text { Mobility: Static and Dynamic } \\
\text { Nature: Cooperative and non-Co-operative }\end{array}$ \\
\hline Operating Environment & Benign Adverse \\
\hline Communication & $\begin{array}{l}\text { Networking: Wired Wireless } \\
\text { Bandwidth: High, Low }\end{array}$ \\
\hline Processing Architecture & Centralized Distributed Hybrid \\
\hline Energy Availability & Constrained and non-constrained \\
\hline
\end{tabular}

Table. 2:Table Type Styles

\begin{tabular}{|c|c|c|c|}
\hline & Yesterday (1980's - 1990's) & Today $(2000-2003)$ & Tomorrow (2010) \\
\hline Manufacturer & $\begin{array}{l}\text { Custom contractors, e.g., for } \\
\text { TRSS }\end{array}$ & $\begin{array}{l}\text { Commercial: Crossbow } \\
\text { Technology, Inc. Sensoria } \\
\text { Corp., Ember Corp. }\end{array}$ & $\begin{array}{l}\text { Dust, Inc. and others to be } \\
\text { formed }\end{array}$ \\
\hline Size & Large shoe box and up & $\begin{array}{l}\text { Pack of cards to small shoe } \\
\text { box }\end{array}$ & Dust particle \\
\hline Weight & Kilograms & Grams & Negligible \\
\hline Node architecture & $\begin{array}{l}\text { Separate sensing, processing } \\
\text { and communication }\end{array}$ & $\begin{array}{l}\text { Integrated sensing. } \\
\text { processing and } \\
\text { communication }\end{array}$ & $\begin{array}{l}\text { Integrated sensing, } \\
\text { processing and } \\
\text { communication }\end{array}$ \\
\hline Topology & Point-to-point, star & Client server, peer to peer & Peer to peer \\
\hline Power supply lifetime & $\begin{array}{l}\text { Large batteries; hours, days } \\
\text { and longer }\end{array}$ & AA batteries; days to weeks & Solar; months to years \\
\hline Deployment & $\begin{array}{l}\text { Vehicle-placed or air-drop } \\
\text { single sensors }\end{array}$ & Hand-emplaced & $\begin{array}{l}\text { Embedded, "sprinkled" left- } \\
\text { behind }\end{array}$ \\
\hline
\end{tabular}

\section{NEW APPLICATIONs}

Research on sensor networks was initially motivated by military applications. The development of communication networks has resulted in developing many other potential applications, from infrastructure security to industrial sensing.

\subsection{Infrastructure Security}

The sensor network is used to secure and protect the critical infrastructure like govt facilitation centre; forces headquarter to protect from terrorist attacks. Video and image sensor, acoustic 
chemical, and biological sensors are used in network form around these facilities [34-39].

\subsection{Environmental and Habitat Monitoring}

The Center for Embedded Net-Work Sensing (CENS) focuses on environmental and ecosystem monitoring. Environmental sensors are used to research the reaction of vegetation to temperature trends and diseases. Acoustic and imaging sensors can recognize, monitor, and bird populations and other animals. For the Amazon Basin, the System for the Vigilance of the Amazon (SIVAM) provides environmental monitoring, drug trafficking monitoring, and air traffic control.

\subsection{Industrial Sensing}

Industries have been gripping in deploying the sensor network to enhance efficiency, machines' performance, and maintenance. They are monitoring the health of the machine by measuring vibration and lubricant levels. Technology (NIST) developed the P1451 Smart Transducer Interface Standard to allow full plugand-play sensors and networks in industrial environments. Factories continued to automate assembly lines and implement advanced on-line quality control checks activated by the sensors. Obtrudes and $\mathrm{pH}$ probes for a right spectral sensor that can serve as miniature spectrometers, optical sensors can replace existing instruments and perform structural properties and composition measurements. Inputs from sensors feed into databases to show real-time information on a large or small scale [40].

\subsection{Traffic Control}

Sensor networks have been used to track and regulate car traffic, sensors overhead, and buried to control and track traffic. When cars approach each other, they share summary information about each other. Ground sensors may create information about traffic jams and the speed and density of traffic. This helps the driver to avoid traffic jams and plan alternative routes.

\section{PROBLEMS AND TECHNICAL CHALLENGES}

Network sensors typically face significant technological challenges in processing data, communication, and sensor management. Due to potentially extreme, unpredictable, and dynamic environments, with energy and bandwidth limitations, ad hoc wireless networks face additional technical difficulties in network discovery, management, and control routing, shared sorting, querying, and tasking [33] .

\subsection{Ad-Hoc Network Discovery}

For an Ad Hoc network, discovery topology must be built-in realtime and modified regularly when sensors fail, or new sensors are installed. Each sensor node interacts with the adjacent sensor. In addition, each sensor knows its location when GPS is not feasible. Relative position algorithm requires for self-location [42].

\subsection{A Network Control and Routing}

The network must deal with energy, bandwidth, and processing power resources that are dynamically evolving, and the device can function independently, changing its configuration as necessary. There is no expected connectivity in ad hoc networks, and connectivity must arise from algorithms and software as required. Since communication links are unstable and shadow fading will erase linkages, the software and device design should produce the necessary reliability. This includes analysis into topics such as the scale of the network or the number of connections and nodes required to ensure sufficient redundancy. Communication gaps and resources need to be well handled. One of the advantages of not having IP addresses at each node is that it is easy to install network devices in vast numbers. Routes are built up from geoinformation on an as-needed basis and optimized for survivability and energy Diffusion routing techniques, which depend only on information in the vicinity of Nodes are a way to fix this, but such approaches do not meet a spatially dispersed wireless network's information-theoretical ability. This is a means of creating relations on demand for data-specific or applicationspecific purposes.

\subsection{Collaborative Signals and Information Processing}

In ad hoc nodes can collaborate to collect and process data to generate the information data. The most technical problem includes the degree to which information exchange data between the nodes and fusion data at nodes. More sensor process data results in better performance and requires more resources. The sensor network is used for the detection, tracking, and classification of the target. If multiple targets are present in a small area, the data association is the primary concern. Each node must associate the measurement with a single target. Targets detected by one node must be aligned with other nodes' targets to prevent replication and allow fusion. Optimal data association is computationally costly and needs a large bandwidth of communication. Processing issue includes latency and reliability and minimizes sensor network life.

\subsection{Tasking and Querying}

A sensor area is like a database with a lot of unique features. Data is dynamically obtained from the environment as opposed to being entered by an operator. Unreliable connections connect geographically scattered nodes; the information is spread across nodes. These features make the database view more complicated for military applications given the low latency, real-time, and high reliability needs of the battlefield. One challenge is to build a querying and tasking language and database that can be quickly queried. Other challenges include discovering effective distributed query and assignment compilation and placement systems, data organization, and caching. Mobile has sensors and query devices. Inter-networking between mobile and fixed devices without any infrastructure crucial and requirement of a sensor network [4346].

\subsection{Security}

Since the sensor network will run in a hostile environment, security should be designed. Network strategies are required to provide low-latency, secure and stable networks. The network should be protected against invasion.

\section{CONCULUSION}

Early research in DNS was obstructed by the state of the art sensors, computers, and communication networks. MEMS technology, more efficient wireless connectivity, and low-cost production have resulted in small, inexpensive, and effective sensors with embedded processing and wireless networking capabilities. The wireless sensor is used in numerous applications in the environment, industry, and military. 


\section{REFERENCES}

[1] Kulkarni, R. N., and C. K. Srinivasa. "Ameliorated Methodology to Meta Model UML Sequence Diagram in the Table Format." Int. J. Advanced Networking and Applications 12, no. 04 (2021): 4633-4638.

[2] Ahmad, T., Li, X. J., \& Seet, B. C. (2017). Parametric loop division for 3D localization in wireless sensor networks. Sensors, 17(7), 1697.

[3] C. E. Nishimura and D. M. Conlon, "IUSS dual use: Monitoring whales and earthquakes using SOSUS," Mar. Technol. Soc. J., vol. 27, no. 4, pp. 13-21, 1994.

[4] Proceedings of the Distributed Sensor Nets Workshop. Pittsburgh, PA: Dept. Comput. Sci., Carnegie Mellon Univ., 1978.

[5] R. F. Sproull and D. Cohen, "High-level protocols," Proc. IEEE, vol. 66, pp. 1371-1386, Nov. 1978.

[6] P. Nii, E. Feigenbaum, J. Anton, and A. Rockmore, "Signalto-symbol transformation: HASP/SIAP case study," AI Mag., vol. 3, pp. 23-36, Spring 1982.

[7] R. R. Smith, "The contract net protocol: High-level communication and control in a distributed problem solver," IEEE Trans. Comput., vol. 29, pp. 1104-1113, Dec.1980.

[8] V. Lesser and D. Corkill, "Functionally accurate, cooperative dis-tributed systems," IEEE Trans. Syst., Man, Cybern., vol. 11, pp. 81-96, Jan./Feb. 1981.

[9] R. B. Wesson, F. A. Hayes-Roth, J. W. Burge, C. Stasz, and C. A. Sunshine, "Network structures for distributed situation assessment," IEEE Trans. Syst., Man, Cybern., vol. SMC-11, pp. 5-23, Jan./Feb. 1981.

[10] R. Rashid and G. Robertson, "Accent: A communication oriented network operating system kernel," in Proc. 8th Symp. Operating System Principles, 1981, pp. 64-75.

[11] R. Rashid, D. Julin, D. Orr, R. Sanzi, R. Baron, A. Forin, D. Golub, and M. Jones, "Mach: A system software kernel," in 34th Computer Society Int. Conf. (COMPCON), San Francisco, CA, 1989.

[12] C. Myers, A. Oppenheim, R. Davis, and W. Dove, "Knowledge-based speec analysis and enhancement," presented at the Int. Conf. Acoustics, Speech and Signal Processing, San Diego, CA, 1984.

[13] S. S. Kulkarni, "TDMA Services for Sensor Networks,"Proc. 24th Int'l. Conf. Distrib. Comp. Sys. Wksps., Mar.2004, pp. 604-09.

[14] W. Ye, J. Heidemann, and D. Estrin, "Medium Access Control with Coordinated Adaptive Sleeping for Wireless Sensor Networks," IEEE/ACM Trans. Net., vol. 12, no. 3, June 2004, pp. 493-506.

[15] A. El-Hoiydi, "Spatial TDMA and CSMA with Preamble Sampling for Low Power Ad Hoc Wireless Sensor Networks," Proc. ISCC 2002, July 2002, pp. 685-92.

[16] V. Rajendran, K. Obraczka, and J. J. Garcia-Luna-Aceves, "Energy-Efficient, Collision-Free Medium Access Control for Wireless Sensor Networks," Proc. ACM SenSys '03, Los Angeles, CA, Nov. 2003, pp. 181-92.

[17] L. Bao and J. J. Garcia-Luna-Aceves, "A New Approach to Channel Access Scheduling for Ad Hoc Networks," 7th Ann. Int'l. Conf. Mobile Comp. and Net., 2001, pp. 210-21.

[18] Ahmad, Tanveer, Xue Jun Li, and Boon-Chong Seet. "A selfcalibrated centroid localization algorithm for indoor ZigBee
WSNs." In 2016 8th IEEE International Conference on Communication Software and Networks (ICCSN), pp. 455461. IEEE, 2016.

[19] Dawadi, B. R., Rawat, D. B., Joshi, S. R., \& Keitsch, M. M. (2020). Towards energy efficiency and green network infrastructure deployment in Nepal using software defined IPv6 network paradigm. The Electronic Journal of Information Systems in Developing Countries, 86(1), e12114.

[20] Ahmad, Tanveer, Xue Jun Li, and Boon-Chong Seet. "3D localization based on parametric loop division and subdivision surfaces for wireless sensor networks." In 2016 25th Wireless and Optical Communication Conference (WOCC), pp. 1-6. IEEE, 2016.

[21] K. Jamieson, H. Balakrishnan, and Y. C. Tay, "Sift: A MAC Protocol for Event-Driven Wireless Sensor Networks," MIT Lab. Comp. Sci., Tech. rep. 894, May 2003, available at http://www.lcs.mit.edu/publications/pubs/pdf/MIT-LCSTR894.pdf

[22] Y. C. Tay, K. Jamieson, and H. Balakrishnan, "CollisionMinimizing CSMA and Its Applications to Wireless Sensor Networks," IEEE JSAC, vol. 22, no. 6, Aug. 2004, pp. 1048-57.

[23] Ahmad, T., Li, X. J., Wenchao, J., \& Ghaffar, A. (2020, September). Frugal Sensing: A Novel approach of Mobile Sensor Network Localization based on Fuzzy-Logic. In Proceedings of the ACM MobiArch 2020 The 15th Workshop on Mobility in the Evolving Internet Architecture (pp. 8-15).

[24] Ahmad, Tanveer, Xue Jun Li, and Boon-Chong Seet. "3D localization using social network analysis for wireless sensor networks." In 2018 IEEE 3rd international conference on communication and information systems (ICCIS), pp. 88-92. IEEE, 2018.

[25] Ahmad, T., Li, X. J., \& Seet, B. C. (2017). Parametric loop division for 3D localization in wireless sensor networks. Sensors, 17(7), 1697.

[26] Chen, Hongyang, Pei Huang, Marcelo Martins, Hing Cheung So, and Kaoru Sezaki. "Novel centroid localization algorithm for three-dimensional wireless sensor networks." In 2008 4th International Conference on Wireless Communications, Networking and Mobile Computing, pp. 1-4. IEEE, 2008.

[27] Chen, Kai, Zhong-hua Wang, Mei Lin, and Min Yu. "An improved DV-Hop localization algorithm for wireless sensor networks." (2010): 255-259.

[28] Ahmad, T. (2019). 3D Localization Techniques for Wireless Sensor Networks (Doctoral dissertation, Auckland University of Technology).

[29] zeng Wang, Ji, and Hongxu Jin. "Improvement on APIT localization algorithms for wireless sensor networks." In 2009 International Conference on Networks Security, Wireless Communications and Trusted Computing, vol. 1, pp. 719-723. IEEE, 2009.

[30] Ahmad, Tanveer, Xue Jun Li, and Boon-Chong Seet. "Noise Reduction Scheme for Parametric Loop Division 3D Wireless Localization Algorithm Based on Extended Kalman Filtering." Journal of Sensor and Actuator Networks 8, no. 2 (2019): 24.

[31] Ahmad, Tanveer, Xue Jun Li, and Boon-Chong Seet. "Fuzzy-Logic Based Localization for Mobile Sensor 


\section{Trends, Technologies, and Future Challenges in Wireless Sensor Network's}

Networks." In 2019 2nd International Conference on Communication, Computing and Digital systems (CCODE), pp. 43-47. IEEE, 2019.

[32] G. Lu, B. Krishnamachari, and C. S. Raghavendra, "An Adaptive Energy-Efficient and Low-Latency MAC for Data Gathering in Wireless Sensor Networks," Proc. 18th Int'1. Parallel and Distrib. Processing Symp., Apr. 2004, p. 224.

[33] Ahmad, Tanveer, Xue Jun Li, Boon-Chong Seet, and JuanCarlos Cano. "Social Network Analysis Based Localization Technique with Clustered Closeness Centrality for 3D Wireless Sensor Networks." Electronics 9, no. 5 (2020): 738.

[34] hakila, R., and B. Paramasivan. "An improved range based localization using Whale Optimization Algorithm in underwater wireless sensor network." Journal of Ambient Intelligence and Humanized Computing (2020): 1-11.

[35] R. Niu and P. Varshney, "Target location estimation in wireless sensor networks using binary data," in Proceedings of the 38th International Conference on Information Sciences and Systems, pp. 17-19, Princeton, NJ, USA, March 2004.

[36] M. P. Michaelides and C. G. Panayiotou, "SNAP: fault tolerant event location estimation in sensor networks using binary data," IEEE Transactions on Computers, vol. 58, no. 9, pp. 1185-1197, 2009.

[37] K. Lu, X. Xiang, D. Zhang, R. Mao, and Y. Feng, "Localization algorithm based on maximum a posteriori in wireless sensor networks," International Journal of Distributed Sensor Networks, vol. 2012, Article ID 260302, 7 pages, 2012.

[38] L. Cheng, C. D. Wu, Y. Z. Zhang, and Y. Wang, "Indoor robot localization based on wireless sensor networks," IEEE Transactions on Consumer Electronics, vol. 57, no. 3, pp. 1099-1104, 2011.

[39] Y. Wang, X. Wang, D. Wang, and D. P. Agrawal, "Rangefree localization using expected hop progress in wireless sensor networks," IEEE Transactions on Parallel and Distributed Systems, vol. 20, no. 10, pp. 1540-1552, 2009.
[40] H. Xu, Y. Tu, W. Xiao, Y. Mao, and T. Shen, “An archimedes curve-based mobile anchor node localization algorithm in wireless sensor networks," in Proceedings of the 8th World Congress on Intelligent Control and Automation (WCICA '10), pp. 6993-6997, Jinan, China, July 2010.

[41] J. Lee, W. Chung, and E. Kim, "Robust DV-Hop algorithm for localization in wireless sensor network," in Proceedings of the International Conference on Control, Automation and Systems, pp. 2506-2509, Gyeonggi-do, South Korea, October 2010.

[42] Ware, R.; Lad, F. Approximating the Distribution for Sum of Product of Normal Variables; Research report; the Mathematics and Statistics department at Canterbury University: Christchurch, New Zealand, 2003.

[43] Wen, C.-Y.; Hsiao, Y.-C. Decentralized anchor-free localization for wireless ad-hoc sensor networks. In Proceedings of IEEE International Conference on Systems, Man, and Cybernetics, Singapore, October 2008; pp. 2777 2785.

[44] Roberts, G.; Gelman, A.; Gilks, W. Weak Convergence and Optimal Scaling of Random Walk Metropolis Algorithms. Technical Report; University of Cambridge: Cambridge, UK, 1994.

[45] Chan, Fu and Chih-yu "Adaptive AoA/ToA localziation using fuzzy particle for mobile WSNs" in 2011 IEEE $73^{\text {rd }}$ VTC Spring 2011.

[46] F. Zhao, J. Shin, and J. Reich, "Information-driven dynamic sensor collaboration for tracking applications," IEEE Signal Processing Mag., vol. 19, pp. 61-72, Mar. 2002. 